\title{
Recomendaciones para el manejo del paciente con enfermedad tiroidea en el escenario de contingencia por COVID-19
}

\section{Recommendations for the management of patients with thyroid disease facing COVID-19}

\author{
Marlen A. Álvarez-Castillo ${ }^{1}$, Raquel N. Faradji-Házan², Michelle N. García-González ${ }^{1}$, \\ Juan C. Garnica-Cueliar ${ }^{3}$, Andrés León-Suarez ${ }^{4}$, Eduardo Márquez-Rodríguez ${ }^{5}$, Alejandro Sosa-Caballero ${ }^{6}$, \\ Rolando ZamarRIPA-ESCOBEDO ${ }^{7}$ y ANTONIO SEGOVIA-PALOMO ${ }^{1 *}$
}

\begin{abstract}
'Departamento de Endocrinología, Clínica de Alta Especialidad en Tiroides, Hospital General de México, Ciudad de México; ${ }^{2}$ Dirección, Clínica EnDi, Ciudad de México; ${ }^{3}$ Departamento de Endocrinología, Centro Médico Nacional 20 Noviembre, Instituto de Seguridad y Servicios Sociales de los Trabajadores del Estado, Ciudad de México; ${ }^{4}$ Servicio de Endocrinología, Hospital Centro Médico ABC, Ciudad de México; ${ }^{5}$ Dirección Médica, Instituto Jalisciense de Metabolismo, Guadalajara, Jalisco; ${ }^{6}$ Departamento de Endocrinología, Centro Médico Nacional La Raza, Instituto Mexicano del Seguro Social, Ciudad de México; ${ }^{7}$ Servicio de Endocrinología, Clínica Dioderm Durango, Durango. México
\end{abstract}

\section{RESUMEN}

Estas recomendaciones tienen como objetivo proporcionar orientación sobre el manejo de alteraciones tiroideas y consideraciones especiales durante la pandemia de enfermedad por coronavirus 2019 (COVID-19). Se han demostrado efectos y la presencia de otros coronavirus en el sistema hipotálamohipófisis-tiroides en epidemias previas, por lo que es posible que el coronavirus 2 del síndrome respiratorio agudo grave (SARS-CoV-2) pueda afectarlos también, aunque hasta el momento la información es limitada. No se recomienda el tamizaje sistemático con perfil tiroideo en pacientes con enfermedad aguda salvo en casos que exista una fuerte sospecha de alteración tiroidea. Siempre que sea posible, hay que valorar la oportunidad de mantener contacto y seguimiento de los pacientes por medio de telemedicina. Los pacientes deben tener suficiente información y conocer datos de alarma, por lo que emitimos estas recomendaciones, mismas que pueden requerir modificaciones de aparecer nueva información.

Palabras clave: COVID-19. Tiroides. SARS-CoV-2. Pandemia.

\begin{abstract}
These recommendations are intended to provide guidance on the management of thyroid dysfunction and special considerations during the coronavirus disease 2019 (COVID-19) pandemic. Effects and the presence of other coronaviruses at the level of the hypothalamic-pituitary-thyroid system have been demonstrated in previous epidemics, so it is possible that severe acute respiratory syndrome coronavirus 2 (SARS-CoV-2) may affect them as well, although until now the information on thyroid repercussions is limited. Routine screening with a thyroid profile is not recommended in patients with acute disease except in cases where there is a strong suspicion of thyroid disorder. Whenever possible, the opportunity to maintain contact and follow-up of patients through telemedicine should be evaluated. Patients should have sufficient information and knowledge of warning signs. We issue these recommendations, which may require modifications if new information were to arise.
\end{abstract}

Key words: COVID-19. Thyroid. SARS-CoV-2. Pandemic.
Correspondencia:

*Antonio Segovia-Palomo

E-mail: sepaan79@hotmail.com
Fecha de recepción: 29-05-2020

Fecha de aceptación: 28-08-2020

DOI: 10.24875/RME.20000062
Disponible en internet: 30-09-2020

Rev Mex Endocrinol Metab Nutr. 2021;8:20-7

2462-4144 / @ 2020 Sociedad Mexicana de Nutricion y Endocrinologia, AC. Publicado por Permanyer. Éste es un artículo open access bajo la licencia CC BY-NC-ND (http://creativecommons.org/licenses/by-nc-nd/4.0/). 


\section{INTRODUCCIÓN}

Las repercusiones sistémicas por coronavirus 2 del síndrome respiratorio agudo grave (SARS-CoV-2) son múltiples ${ }^{1}$.

Se reportó, en una serie de 50 casos, niveles significativamente más bajos de hormona estimulante de la tiroides (TSH) y de triyodotironina total durante las manifestaciones de enfermedad por coronavirus 2019 (COVID-19) y normalización de estos al recuperarse $^{2}$. Sin embargo, los cambios en los niveles de las hormonas tiroideas también pueden estar relacionados con la presencia del síndrome del eutiroideo enfermo o disfunción tiroidea del enfermo grave, por lo que es importante, en caso de realizar un perfil tiroideo, la correcta interpretación de estas alteraciones ${ }^{3}$. Si bien las repercusiones actualmente conocidas del SARS-CoV-2 sobre el sistema tiroideo son limitadas, hay varias situaciones del manejo de la patología tiroidea que requieren de observaciones o recomendaciones durante la emergencia sanitaria.

Esta información puede cambiar de aparecer nueva información.

\section{SISTEMA TIROIDEO Y SÍNDROME RESPIRATORIO AGUDO SEVERO}

Se han propuesto dos mecanismos para el ingreso del SARS-CoV-2 al sistema nervioso central, ya sea por la afectación de los pares craneales del olfato y del gusto pasando la placa cribiforme y/o hematógena directa ${ }^{4}$.

El tejido hipotalámico e hipofisario expresan la enzima convertidora de angiotensina $2^{4,5}$.

En la epidemia previa de otro coronavirus, se identificó en necropsias edema, degeneración neuronal y genoma del síndrome respiratorio agudo severo (SARS) a nivel del hipotálamo e hipófisis ${ }^{4}$.

También se documentaron afectaciones funcionales del eje hipotálamo-hipófisis asociadas a hipocortisolismo central en el $40 \%$ de los casos, la mayoría de los cuales se normalizó dentro del año de presentación (62.5\%), e hipotiroidismo central en el $5 \%$ de los $\operatorname{casos}^{6}$.
En el 2007 se reportó, en una pequeña serie de necropsias, el daño estructural que sufre el tejido tiroideo (folicular y parafolicular) por efecto del coronavirus causante de la epidemia de SARS entre 2002-2004 y se ha discutido sobre el potencial efecto en la producción de las hormonas tiroideas y de la calcitonina ${ }^{7,8}$.

La disminución en los niveles de calcitonina es un mecanismo plausible de la osteonecrosis de la cabeza femoral observada en pacientes recuperados de $\mathrm{SARS}^{4,7}$.

\section{Recomendación 1. En la toma de perfil tiroideo de paciente con COVID-19:}

- No se recomienda el tamizaje sistemático para la disfunción tiroidea en pacientes con enfermedades agudas a menos que haya una fuerte sospecha de que la enfermedad tiroidea está contribuyendo a la presentación clínica ${ }^{5,9}$.

\section{HIPOTIROIDISMO}

El tratamiento con levotiroxina es el recomendado en general para el hipotiroidismo. Con él se busca resolver los datos clínicos de hipotiroidismo y normalizar las pruebas de funcionamiento tiroideo. Cuando se está ajustando la dosis se recomienda realizar estudio de control en cuatro a seis semanas 9 .

Recomendación 2. En caso del tratamiento del hipotiroidismo:

- Los pacientes que ya reciben tratamiento y se encuentran estables pueden continuar con la misma dosificación.

- Recomendamos tener suficiente medicamento en casa si se encuentra con una dosis estable, hasta para cuatro a seis meses ${ }^{5}$.

- En caso de hospitalización con hipotiroidismo controlado y tolerancia a la vía oral, se recomienda mantener la misma dosis de levotiroxina. En 
aquellos con intolerancia a la vía oral esta puede ser administrada por vía enteral o, de contar con la administración intravenosa, se recomienda ajustar al $75 \%$ de la dosis usualmente requerida 9 .

Recomendación 3. En caso de perfil tiroideo de seguimiento o diagnóstico:

- Realizar el perfil tiroideo durante la contingencia se recomienda para aquellos pacientes en proceso de ajuste de dosis, embarazadas o pacientes sintomáticos.

- Se sugiere localizar laboratorios que incluyan toma a domicilio a fin de evitar riesgos asociados a exposición y traslado a laboratorios.

- Se debe continuar realizando el tamiz neonatal y tener los datos de localización con la finalidad de iniciar un tratamiento oportuno y evitar secuelas del hipotiroidismo congénito ${ }^{10,11}$.

\section{TIROIDITIS}

La tiroiditis subaguda dolorosa está generalmente precedida por procesos infecciosos de la vía aérea superior $^{12}$.

Brancatella recientemente reportó el primer caso de tiroiditis subaguda en un paciente con COVID-19 ${ }^{13}$.

\section{Recomendación 4. En el caso de sospecha} de tiroiditis subaguda:

- De tener manifestaciones de tirotoxicosis semanas después del diagnóstico de COVID-19, hay que descartar la presencia de tiroiditis subaguda.

\section{HIPERTIROIDISMO}

Los pacientes tratados con tionamida (tiamazol, carbimazol, propiltiouracilo) pueden presentar agranulocitosis; efecto secundario poco común (0.2-0.5\%), pero que puede dar síntomas como fiebre, úlceras orales y odinofagia, con neutropenia ${ }^{14-16}$.

Esto último es un factor de riesgo para contraer infecciones, incluido el SARS-CoV-2, y de sobreinfección bacteriana, lo que condicionaría una expresión clínica más severa ${ }^{5,17}$.

\section{Recomendación 5. En el caso de uso de tionamida:}

- En caso de uso de tionamida con presencia de fiebre y/o odinofagia, hacer diagnóstico diferencial para excluir infección por SARS-CoV- $2^{5}$.

- Se debe explicar a los pacientes para identificar datos de riesgo del uso de tionamida y buscar atención médica inmediata ${ }^{5}$.

- En caso de descartar agranulocitosis por tionamida se sugiere reiniciar con una dosis más baja de tiamazol y en caso de recurrir con neutropenia se debe considerar el empleo de otras alternativas terapéuticas ${ }^{16}$.

- En caso de contraindicación para uso de tionami$\mathrm{da}$, preparar al paciente para tratamiento definitivo del hipertiroidismo con radioyodo o cirugía; el tiempo para llevarlo a cabo depende de la severidad de la tirotoxicosis y del riesgo de complicaciones $^{5,18-20}$.

- En aquellos pacientes tratados con tionamida y hospitalizados por COVID-19, se puede continuar la administración, vigilando las cifras de neutrófilos. En los que la administración oral del medicamento no sea factible, este puede ser administrado por medio de sonda nasogástrica o por vía rectal y, cuando se encuentre disponible, por vía intravenosa $a^{9,16}$.

\section{OFTALMOPATÍA TIROIDEA}

Algunos pacientes con oftalmopatía tiroidea llevan tratamiento con glucocorticoides a dosis que inhiben el sistema inmunitario o con otros fármacos inmunosupresores, como micofenolato, rituximab, 
tocilizumab, metotrexato o azatioprina; el uso de estos medicamentos conlleva al incremento del riesgo de infecciones ${ }^{21,22}$.

EI SARS-CoV-2 ha sido aislado en lágrima y la conjuntivitis es una de las afectaciones reportadas en personas con COVID-1923.

\section{Recomendación 6. En caso de conjuntivitis como manifestación de COVID-19:}

- En caso de pacientes con enfermedad de Graves o sospecha de esta que presentan afectación orbitaria, descartar infección por SARS-CoV-2 $2^{5}$.

Recomendación 7. En el uso de esteroides y/o de otros tratamientos inmunosupresores para orbitopatía de Graves:

- Suspender tabaquismo en caso de este, medidas de protección ocular como gafas obscuras en caso de exponerse a luz solar o polvo, antifaz al dormir en caso de falta de oclusión palpebral. Mantener el confinamiento siempre que sea posible ${ }^{5,20}$.

- Aquellos pacientes que presenten nuevos síntomas, progresión de la orbitopatía tiroidea o que no estén respondiendo al tratamiento establecido deben ser revalorados.

\section{ENFERMEDAD NODULAR}

La mayoría de los nódulos tiroideos son benignos, pero entre el 5 y el $15 \%$ son malignos. Por lo que, de acuerdo con las características clínicas y ultrasonográficas basadas en TI-RADS (Thyroid Imaging Reporting and Data System), se aconseja realizar una biopsia por aspiración ${ }^{24-27}$.

Si bien diferenciar un nódulo neoplásico de uno benigno es esencial para guiar el manejo clínico, es poco probable que esperar algunos meses en el diagnóstico afecte negativamente al resultado en la mayoría de los pacientes, pero deben considerarse las manifestaciones clínicas y los factores de riesgo.
Recomendación 8. En la realización de biopsia por nódulo tiroideo:

- Se recomienda posponer la biopsia de la mayoría de los nódulos tiroideos asintomáticos, con el consentimiento del paciente ${ }^{28}$.

- Es recomendable realizar la biopsia cuando hay presencia de síntomas significativos y/o signos de compresión, como crecimiento rápido, adenopatías de cuello sospechosas por ultrasonido, parálisis sospechosa de las cuerdas vocales y/o disfagia $^{29}$. También en casos con sospecha de cáncer medular por antecedente de historia familiar, localización en polo superior o calcitonina $>20$ pero $<100 \mathrm{pg} / \mathrm{ml}^{29,30}$. Así como en los pacientes pediátricos en estudio por adenopatías sospechosas e identificación de nódulo tiroideo ${ }^{28}$.

- La biopsia de tiroides puede considerarse de riesgo debido a que se han demostrado efectos directos del SARS en este tejido y al proceso de aspersión de células en las laminillas ${ }^{8,19}$.

- En caso de realizar biopsia, extremar medidas de precaución con el equipo de protección personal para riesgo de procedimientos con aerosoles (gafas o careta protectora, respirador N95, bata y guantes) ${ }^{27,30,31}$.

- Es preferible estar solo el personal indispensable para la realización de la biopsia, por lo que en general se recomienda que no esté presente el patólogo y se envíe la muestra al laboratorio para disminuir el número de personal potencialmente expuesto ${ }^{27}$.

- Al esparcir la muestra sobre las laminillas se debe hacer con la mínima presión sobre el embolo de la jeringa para no generar aerosol. Una alternativa puede ser tomar las muestras solo con aguja y realizar extendido directo del capuchón hacia la laminilla. La laminilla se debe colocar rápidamente en la solución fijadora con alcohol etílico al 96\%.

\section{CÁNCER DIFERENCIADO DE TIROIDES}

La mayoría de los carcinomas tiroideos son diferenciados (papilar y folicular), los cuales no se han 
asociado con mayor riesgo de desarrollar un curso de COVID-19 severo, pero debe tenerse en cuenta que los pacientes oncológicos en general sufren un riesgo 3.5 veces mayor de ventilación mecánica, de ingreso a unidades de cuidados intensivos o de muerte en comparación con los pacientes sin cán$\operatorname{cer}^{28,32}$.

Los Centros para el Control de Enfermedades de E.U.A. consideran que los pacientes que reciben tratamientos oncológicos cumplen con la definición de inmunocomprometidos; sin embargo, a diferencia de muchos otros tipos de cáncer, la mayoría de los pacientes con cáncer de tiroides no reciben quimioterapia u otro tratamiento que comprometa el sistema inmunitario; en ese sentido, tener un diagnóstico previo de cáncer de tiroides y recibir hormona tiroidea no es un factor de riesgo conocido para contraer SARS-CoV-2 o presentar la manifestación más grave de COVID-1929.

Diferentes sociedades médicas quirúrgicas ${ }^{13}$ han recomendado evitar cirugías electivas durante la fase de contingencia de SARS-CoV-2 ${ }^{33-36}$.

La tiroidectomía representa el tratamiento de primera línea para los pacientes con carcinoma diferenciado de tiroides, pero su diferimiento por tres a seis meses no parece contribuir a un mayor riesgo de proliferación o progresión en la mayoría de los pacientes, que son de bajo riesgo por evaluación clínica debido a que la mayoría son de crecimiento lento ${ }^{14,38}$. Incluso los microcarcinomas pueden entrar en protocolo de vigilancia estrecha ${ }^{37-39}$.

De tal forma que el tratamiento quirúrgico debe reservarse a pacientes con tumores grandes y/o que condicionen síntomas por efecto vector, tumores de crecimiento rápido y formas agresivas del carcinoma tiroideo, incluyendo pacientes con cáncer diferenciado con enfermedad metastásica pulmonar grave cuyo pronóstico puede cambiar con las intervenciones, cáncer poco diferenciado, el anaplásico o el medular tiroideo ${ }^{13}$; también en pacientes pediátricos con progresión ${ }^{28,35}$ y en aquellos de alto riesgo con recidiva después de una cuidadosa evaluación de riesgo-beneficio ${ }^{28}$.

El tratamiento con yodo radioactivo en sus diferentes modalidades (ablativo, adyuvante) ha sido suspendido en algunos centros. Se considera que un retraso en su administración de alrededor de seis meses no afecta negativamente al pronóstico del cáncer diferenciado en la mayoría de los casos, que son de bajo riesgo ${ }^{29,40}$.

En aquellos pacientes con enfermedad metastásica fuera de cuello o enfermedad progresiva se puede presentar un escenario de mayor necesidad de administración del tratamiento con yodo radioactivo; en estos casos se recomienda evaluar de manera individual el riesgo-beneficio, preferentemente de manera multidisciplinaria, y tomar las medidas pertinentes. Continuar, si es posible, la administración en aquellos que están ya en preparación para recibir el radioyodo o que están con agentes rediferenciadores para su aplicación ${ }^{28}$.

Los pacientes con cáncer tiroideo refractario a radioyodo, carcinoma anaplásico o carcinoma medular que reciben quimioterapia o inhibidores de tirosina cinasa (lenvatinib, sorafenib, vandetanib, etc.) son de mayor riesgo para presentar estados severos de COVID-1931,41,42.

Además, los pacientes que previamente recibieron radioterapia de haz externo en el cuello, también pueden tener un mayor riesgo de presentaciones severas de COVID-1943.

\section{Recomendación 9. En caso del tratamiento del cáncer de tiroides:}

- Valorar en equipo multidisciplinario el estadio clínico y pronóstico del paciente con carcinoma diferenciado de tiroides para decidir el tiempo de espera para cirugía o la necesidad de intervención.

- Implementar protocolos de vigilancia activa para el seguimiento de microcarcinomas.

- En medida de lo posible, recomendamos que aquellos que ya se encuentran en protocolo para aplicación de radioyodo con suspensión de levotiroxina o con agentes rediferenciadores, reciban el radioyodo con las medidas de protección necesarias para disminuir riesgos de contagio y con el consentimiento del paciente (en 
caso de no ser posible reprogramar y reiniciar el protocolo de preparación cuando sea más seguro).

- En aquellos casos en los que la administración de radioyodo se considera que puede modificar la evolución de la enfermedad, recomendamos tomar la decisión en un equipo (de preferencia multidisciplinario) del tiempo adecuado y de ser posible, el más seguro para la aplicación de este.

- Para los que se encuentra con quimioterapia, con uso de inhibidores de tirosina cinasa o en tratamiento de radioterapia externa, se deben considerar de mayor riesgo para contagio y para presentar mayor severidad de COVID-19, por lo que recomendamos mantener estrechamente las medidas generales de protección y confinamiento.

- En caso de cirugía, se deben tomar todas las medidas de protección universal, incluyendo el uso del equipo de protección personal; adicionalmente, es preferible contar solo con el personal indispensable para disminuir el número de personas potencialmente expuestas.

EMBARAZO Y ENFERMEDAD TIROIDEA

Las mujeres embarazadas con infección por SARSCoV-2 presentan grados variables de la enferme$\operatorname{dad}^{44,45}$.

Las embarazadas con hipotiroidismo deben de seguir las mismas recomendaciones de realizar su perfil tiroideo para valorar ajustes en la dosis de levotiroxina una vez que tengan una prueba de embarazo positiva y cada cuatro semanas durante la primera mitad del embarazo ${ }^{46}$, y en el resto hacerlo cada cuatro a ocho semanas con base en la evolución del embarazo.

Idealmente se debe vigilar el perfil tiroideo de una manera regular buscando mantenerse en los valores establecidos por trimestre en embarazo de contar con las referencias para la población tratada por hipotiroidismo primario y cuando no se dispone de ellos, buscar mantener márgenes de la
$\mathrm{TSH}<2.5 \mathrm{mUl} / \mathrm{ml}$ en el límite superior y normal en el límite inferior ${ }^{46,47}$.

En la mujer embarazada con hipertiroidismo se recomienda utilizar antitiroideos a la dosis mínima posible y realizar su perfil tiroideo cada cuatro semanas, buscando mantener los niveles de tiroxina total y/o libre cercanos al límite superior normal ${ }^{14,16,46}$.

\section{Recomendación 10. En caso de embarazo $y$ alteraciones del funcionamiento tiroideo:}

- La embarazada con hipotiroidismo o hipertiroidismo en tratamiento debe realizar regularmente su perfil tiroideo para valorar encontrarse en metas de tratamiento o hacer los ajustes necesarios

- En caso de hipotiroidismo, mantener contacto con su médico. Si es posible, por medio electrónico y cuando se requiera, acudir a su revisión.

- Cuando se está en seguimiento por hipertiroidismo, recomendamos acudir a sus revisiones de vigilancia materno-fetal, con adecuadas medidas de seguridad para reducir el riesgo de contagio.

- Deben ser informadas de los datos de alarma de los tratamientos para buscar atención médica en caso necesario.

\section{TELEMEDICINA Y ENFERMEDAD TIROIDEA}

En las actuales circunstancias de limitación de contacto social para evitar la propagación del COVID-19 ${ }^{48}$.

\section{Recomendación 11. Para el uso de telemedicina en patologías tiroideas:}

- Para la mayoría de las consultas relacionadas con trastornos tiroideos, en particular en el seguimiento de hipotiroidismo, vigilancia de pacientes estables con hipertiroidismo, nódulos tiroideos o cáncer de tiroides estables y asintomáticos. La atención y orientación pueden ser modificadas a 
llamadas telefónicas, videollamadas o correo electrónico, siguiendo la normativa establecida para cualquier tipo de atención médica, incluida la virtual $\left.\right|^{5,15,28,49-50}$.

- Recomendamos mantener un método para lograr una atención virtual organizada, utilizando métodos como el PSOAP (generalidades, padecimientos, medicamentos del paciente; subjetivo, síntomas y necesidades del paciente; objetivo: datos posibles de la exploración física, estudios de laboratorio, registros; apreciación para establecer diagnóstico(s); plan de manejo, recetas, indicaciones y acuerdos a seguir) ${ }^{51}$.

\section{URGENCIAS TIROIDEAS}

Existen urgencias tiroideas que deben ser atendidas en forma hospitalaria, como la tormenta tiroidea y el coma mixedematoso, así como oportunas, en caso del hipotiroidismo congénito.

\section{Recomendación 12. En caso de urgencias} tiroideas:

Recomendamos se mantengan los protocolos de atención para pacientes que requieren atención de urgencia o de hospitalización para el control de los padecimientos tiroideos que ponen en peligro la vida o algún órgano.

\section{CONCLUSIONES}

Se requieren estudios para evaluar los efectos directos del SARS-CoV-2 sobre el tejido tiroideo y el eje de funcionamiento tiroideo, así como el impacto clínico que esto pueda representar.

Emitimos estas recomendaciones respecto a algunas situaciones que pudiesen presentarse en la atención de pacientes con patología tiroidea, mismas que pueden requerir modificarse de aparecer información nueva.

\section{FINANCIAMIENTO}

La presente investigación no ha recibido ayudas específicas provenientes de agencias del sector público, sector comercial o entidades sin ánimo de lucro.

CONFLICTO DE INTERESES

Los autores declaran no tener conflicto de intereses alguno.

\section{RESPONSABILIDADES ÉTICAS}

Protección de personas y animales. Los autores declaran que para esta investigación no se han realizado experimentos en seres humanos ni en animales.

Confidencialidad de los datos. Los autores declaran que han seguido los protocolos de su centro de trabajo sobre la publicación de datos de pacientes.

Derecho a la privacidad y consentimiento informado. Los autores declaran que en este artículo no aparecen datos de pacientes.

\section{BIBLIOGRAFÍA}

1. Wu Z, McGoogan JM. Characteristics of and important lessons from the coronavirus disease 2019 (COVID-19) outbreak in China: Summary of a report of 72314 cases from the Chinese Center for Disease Control and Prevention. JAMA. 2020;323(13):1239-42.

2. Chen M, Zhou W, Xu W. Thyroid function analysis in 50 patients with COVID-19: A retrospective study. Thyroid. 2020;10.1089/thy.2020.0363. doi:10.1089/thy.2020.0363. Online ahead of print.

3. Lee S, Farwell AP. Euthyroid sick syndrome. Compr Physiol. 2016;6(2):1071-80.

4. Pal R, Banerjee M. COVID-19 and the endocrine system: exploring the unexplored. J Endocrinol Invest. 2020;43(7):1027-31.

5. Boelaert K, Visser WE, Taylor PN, Moran C, Leger J, Persani L. Endocrinology in the time of COVID-19: Management of hyperthyroidism and hypothyroidism. Eur J Endocrinol. 2020;183(1):G33-G39.

6. Leow MKS, Kwek DSK, Ng AWK, Ong KC, Kaw GJL, Lee LS. Hypocortisolism in survivors of severe acute respiratory syndrome (SARS). Clin Endocrinol. 2005:63(2):197-202.

7. Wei L, Sun S, Xu CH, Zhang J, Xu Y, Zhu H, et al. Pathology of the thyroid in severe acute respiratory syndrome. Hum Pathol. 2007;38(1):95-102.

8. Pantanowitz L. Handling of thyroid FNA samples during the COVID-19 pandemic. Clin Thyroidol. 2020; 32(5):239-41. 
9. Jonklaas J, Bianco AC, Bauer AJ, Burman KD, Cappola AR, Celi FS, et al Guidelines for the treatment of hypothyroidism: prepared by the American Thyroid Association task force on thyroid hormone replacement. Thyroid. 2014:24(12):1670-751.

10. NORMA Oficial Mexicana NOM-034-SSA2-2002, Para la prevención y control de los defectos al nacimiento [Internet]. México: Gobierno de México [fecha de consulta: 16 de julio de 2020]. Disponible en: http:// www.salud.gob.mx/unidades/cdi/nom/034ssa202.html

11. Léger J, Olivieri A, Donaldson $M$, Torresani T, Krude H, et al.; Congenital Hypothyroidism Consensus Conference Group. Congenital Hypothyroidism Consensus Conference Group, European Society for Pediatric Endocrinology consensus guidelines on screening, diagnosis, and management of congenital hypothyroidism. J Clin Endocrinol Metab. 2014;99(Suppl 2):363-84.

12. Desailloud R, Hober D. Viruses and thyroiditis: an update. Virol J. 2009;6(1):5.

13. Brancatella A, Ricci D, Viola N, Sgrò D, Santini F, Latrofa F. Subacute thyroiditis after SARS-CoV-2 infection. J Clin Endocrinol Metab. 2020;105(7):dgaa276.

14. Ross DS, Burch HB, Cooper DS, Greenlee MC, Laurberg P, Maia AL, et al. 2016 American Thyroid Association guidelines for diagnosis and management of hyperthyroidism and other causes of thyrotoxicosis. Thyroid. 2061;26(10):1343-421.

15. Curtis BR. Non-chemotherapy drug-induced neutropenia: key points to manage the challenges. Hematology Am Soc Hematol Educ Program. 2017;2017(1):187-93.

16. Burch HB, Cooper DS. Antithyroid drug therapy: 70 years later. Eur J Endocrinol. 2018;179(5):R261-R274.

17. Kalil AC. Treating COVID-19-Off-label drug use, compassionate use, and randomized clinical trials during pandemics. JAMA. 2020;323(19):1897-8.

18. Zhou F, Yu T, Du R, Fan G, Liu Y, Liu Z, et al. Clinical course and risk factors for mortality of adult inpatients with COVID-19 in Wuhan, China: a retrospective cohort study. Lancet. 2020;395:1054-62.

19. COVID-19 resources for managing endocrine conditions [Internet]. Reino Unido: Society for Endocrinology [fecha de consulta: 16 de julio de 2020]. Disponible en: https://www.endocrinology.org/clinical-practice/ covid-19-resources-for-managing-endocrine-conditions/

20. Kahaly GJ, Bartalena L, Hegedüs L, Leenhardt L, Poppe K, Pearce SH. 2018 European thyroid association guideline for the management of Graves' hyperthyroidism. Eur Thyroid J. 2018;7(4):167-86.

21. Taylor PN, Zhang L, Lee RW, Muller I, Ezra DG, Dayan CM, et al. New insights into the pathogenesis and nonsurgical management of Graves orbitopathy. Nat Rev Endocrinol. 2020;16(2):104-16.

22. COVID-19: guidance on shielding and protecting people defined on medical grounds as extremely vulnerable [Internet]. Reino Unido: Public Health England [fecha de consulta: 16 de julio de 2020]. Disponible en: https://www.gov.uk/government/publications/guidance-on-shieldingand-protecting-extremely-vulnerable-persons-from-covid-19/

23. Xia J, Tong J, Liu M, Shen Y, Guo D. Evaluation of coronavirus in tears and conjunctival secretions of patients with SARS-CoV-2 infection. J Med Virol. 2020;92(6):589-94.

24. Durante C, Grani G, Lamartina L, Filetti S, Mandel SJ, CooperDS. The diagnosis and management of thyroid nodules: a review. JAMA. 2018;319:914-24

25. US Preventive Services Task Force, Bibbins-Domingo K, Grossman DC, Curry SJ, Davidson KW, Epling JW, García FAR, et al. Screening for colorectal cancer: US Preventive Services Task Force recommendation statement. JAMA. 2016;315(23):2564-75.

26. Tessler FN, Middleton WD, Grant EG, Hoang JK, Berland L, Teefey SA, et al. ACR thyroid imaging, reporting and data system (TI-RADS): white paper of the ACR TI-RADS committee. J Am Coll Radiol. 2017;14(5):587-95.

27. Li M, Marquez RC, Vinales KL, Reaven PD, Behari G, Dildeep A, et al. Considerations for thyroid fine needle aspiration (FNA) biopsies during the COVID-19 pandemic. Clin Thyroidol. 2020;32:156-58.

28. Vrachimis A, lakovou I, Giannoula E, Giovanella L. Endocrinology in the time of COVID-19: Management of thyroid nodules and cancer. Eur J Endocrinol. 2020;183(1):G41-G48.

29. Haugen BR, Alexander EK, Bible KC, Doherty GM, Mandel SJ, Nikiforov, et al. 2015 American Thyroid Association management guidelines for adult patients with thyroid nodules and differentiated thyroid cancer: the American Thyroid Association guidelines task force on thyroid nodules and differentiated thyroid cancer. Thyroid. 2016;26(1):1-133.

30. Elisei R, Bottici V, Luchetti F, DiCoscio G, Romei C, Grasso L, et al. An impact of routine measurement of serum calcitonin on the diagnosis and outcome of medullary thyroid cancer: experience in 10,864 patients with nodular thyroid disorders. J Clin Endocrinol Metab. 2004;89:163-8.
31. BAETS statement on COVID-19 and Thyroid Cancer Services. Confidential advice for health professionals to consider when planning thyroid cancer surgical services [Internet]. British Association of Endocrine \& Thyroid Surgeons [fecha de consulta: 15 de mayo de 2020]. Disponible en: https://www.baets.org.uk/wp-content/uploads/2020/05/BAETS Statement-Thyroid-Cancer-Covid-0520.pdf

32. Liang W, Guan W, Chen R, Wang W, Li J, Xu K, et al. Cancer patients in SARS-CoV-2 infection: a nationwide analysis in China. Lancet Oncol. 2020;21:335-7.

33. Commins J. Surgeon general urges providers to "consider stopping all elective surgeries". Hospitals push back [Internet]. Health Leaders [fecha de consulta: 17 de marzo de 2020]. Disponible en: https://www. healthleadersmedia.com/clinical-care/surgeon-general-urges-providers-consider-stopping-elective-surgeries-hospitals-push

34. COVID-19 update: guidance for triage of non-emergent surgical procedures [Internet]. American College of Surgeons [fecha de consulta: 17 de marzo de 2020]. Disponible en: https://www.facs.org/covid-19/clinical-guidance/triage

35. Al-Omar K, Bakkar S, Khasawneh L, Donatini G, Miccoli P. Resuming elective surgery in the time of COVID-19: a safe and comprehensive strategy. Updates Surg. 2020;72(2):291-5

36. Bakkar S, Al-Omar K, Aljarrah Q, Al-Dabbas M, Al-Dabbas N, Samara S, et al. Impact of COVID-19 on thyroid cancer surgery and adjunct therapy. Updates Surg. 2020 Jun 14;1-3. doi: 10.1007/s13304-020-00833-3. Online ahead of print.

37. Shaha AR. Thyroid surgery during COVID-19 pandemic: Principles and philosophies. Head Neck. 2020;42(6):1322-4.

38. Miyauchi A. Clinical trials of active surveillance of papillary microcarcinoma of the thyroid. World J Surg. 2016;40(3):516-22.

39. Tuttle RM, Fagin JA, Minkowitz G, Wong RJ, Roman B, Patel S, et al. Natural history and tumor volume kinetics of papillary thyroid cancers during active surveillance. JAMA Otolaryngol Head Neck Surg. 2017;143:1015-20.

40. Wadsley J, Moss L. Thyroid Cancer: Radioactive lodine Treatment during COVID19 pandemic [Internet]. Reino Unido: Society for Endocrinology [fecha de consulta: 16 de julio de 2020]. Disponible en: https://www. endocrinology.org/media/3575/radioactive-iodine-treatment-duringcovid19-pandemic.pdf

41. Ancker OV, Kruger $M$, Wehland $M$, Infanger $M$, GrimmD. Multikinase inhibitor treatment in thyroid cancer. Int J Mol Sci. 2019;21(1):10.

42. ETA Public Health Board Statement: COVID-19: information and recommendations for patients with thyroid diseases [Internet]. European Thyroid Association [fecha de consulta: 4 de mayo de 2020]. Disponible en: https://www.eurothyroid.com/files/download/ETA-PHB.pdf

43. Romesser PB, Cahlon O, Scher E, Zhou Y, Berry SL, Rybkim A, et al. Proton beam radiation therapy results in significantly reduced toxicity compared with intensity-modulated radiation therapy for head and neck tumors that require ipsilateral radiation. Radiother Oncol. 2016;118:286-92.

44. Deprest J, Choolani M, Chervenak F, Farmer D, Lagrou K, Lopriore E, et al. Fetal diagnosis and therapy during the COVID-19 pandemic: guidance on behalf of the International Fetal Medicine and Surgery Society. Fetal Diagn Ther. 2020;47(9):689-98.

45. Qiao, J. What are the risks of COVID-19 infection in pregnant women? Lancet. 2020;395(10226):760-2.

46. Alexander EK, Pearce EN, Brent GA, Brown RS, Chen H, Dosiou C, et al. 2017 Guidelines of the American Thyroid Association for the diagnosis and management of thyroid disease during pregnancy and the postpartum. Thyroid. 2017;27(3):315-89.

47. Pearce,E.N A comparison of ATA and Updated ACOG Guidelines for Thyroid Disease in Pregnancy. Clinical Thyroidology. 2020; 32: 317-320.

48. https://www.gmc-uk.org/ethical-guidance/ethical-hub/remote-consultations. Consultado el 16 de julio 2020.

49. Cédula de Instrumentos Jurídicos aplicables a la práctica de la Telesalud en México [Internet]. México: Secretaría de Salud, Centro Nacional de Excelencia Tecnológica en Salud; 2019 [fecha de consulta: 23 de julio de 2020]. Disponible en: https://www.gob.mx/salud/cenetec/documentos/ cedula-de-instrumentos-juridicos-aplicables-a-la-practica-de-la-telesalud-en-mexico

50. Marcos Normativos para el uso de la Telesalud [Internet]. México: Secretaría de Salud; 2015 [fecha de consulta: 23 de julio de 2020]. Disponible en:. http://www.cenetec.salud.gob.mx/contenidos/telesalud/marco_ norma.html

51. Faradji-Hazán RN, Madrigal-Sanromán JR, Sainz de la Maza-Viadero ME, Márquez-Rodríguez E. La pandemia de COVID-19 ha impulsado el manejo de la diabetes vía telemedicina. Rev Mex Endocrinol Metab Nutr. 2020;7:87-95 